\title{
Democracy in Hard Times: \\ Economic Shocks, Social Capital and Voting Patterns
}

\author{
Francesc Amat ${ }^{1}$
}

${ }^{1}$ University of Barcelona \& IPERG

APSA Conference 2020 Online Meeting

September 2020 


\section{Motivation}

This paper investigate how contemporaneous negative economic shocks interact with latent and pre-existing cultural roots: 


\section{Motivation}

This paper investigate how contemporaneous negative economic shocks interact with latent and pre-existing cultural roots:

1 Intends to go further than discussions that separate economic explanations from cultural ones 


\section{Motivation}

This paper investigate how contemporaneous negative economic shocks interact with latent and pre-existing cultural roots:

1 Intends to go further than discussions that separate economic explanations from cultural ones

2 Approaches the exploration of current unemployment shocks by exploiting a new dataset on layoffs at the regional-level 


\section{Motivation}

This paper investigate how contemporaneous negative economic shocks interact with latent and pre-existing cultural roots:

1 Intends to go further than discussions that separate economic explanations from cultural ones

2 Approaches the exploration of current unemployment shocks by exploiting a new dataset on layoffs at the regional-level

3 Explores the role of social capital as the mediating variable that can explain the persistence of deep cultural roots 


\section{Preview}

The results suggest that historical family types condition the effects of current unemployment shocks on voting patterns and political trust 


\section{Preview}

The results suggest that historical family types condition the effects of current unemployment shocks on voting patterns and political trust

Stem family regions alleviate the effect of unemployment layoffs on nationalist voting (reducing, however, political trust) 


\section{Preview}

The results suggest that historical family types condition the effects of current unemployment shocks on voting patterns and political trust

Stem family regions alleviate the effect of unemployment layoffs on nationalist voting (reducing, however, political trust)

Communitarian and egalitarian nuclear family regions, instead, amplify nationalist voting and increase political trust 


\section{Preview}

The results suggest that historical family types condition the effects of current unemployment shocks on voting patterns and political trust

Stem family regions alleviate the effect of unemployment layoffs on nationalist voting (reducing, however, political trust)

Communitarian and egalitarian nuclear family regions, instead, amplify nationalist voting and increase political trust

Also evidence for the mechanism: 


\section{Preview}

The results suggest that historical family types condition the effects of current unemployment shocks on voting patterns and political trust

Stem family regions alleviate the effect of unemployment layoffs on nationalist voting (reducing, however, political trust)

Communitarian and egalitarian nuclear family regions, instead, amplify nationalist voting and increase political trust

Also evidence for the mechanism:

- Social capital as the intervening factor that links historical family types, contemporaneous shocks and political responses

- Documents a reversal of the effect of social capital on nationalist voting 


\section{Theory I: Literature review}

- Long standing literature on the effect of family types on in-group and out-gorup trust, institutional quality and development (Alesina and Giuliano 2010, 2014; Grief 2005; Duranton et al 2009; Hager and Hilbig 2019; Tur-Prats 2019). 


\section{Theory I: Literature review}

- Long standing literature on the effect of family types on in-group and out-gorup trust, institutional quality and development (Alesina and Giuliano 2010, 2014; Grief 2005; Duranton et al 2009; Hager and Hilbig 2019; Tur-Prats 2019).

- Less studies on the political consequences of family types (Todd 1985, 1987, 1990). And even lesson the mechanisms of persistence of historical family types (Hager and Hilbig 2019, Tur-Prats 2020, Giuliano and Nunn 2017). 


\section{Theory II: Literature review}

- Combining Banfield (1958) and Coleman (1988) arguments, 


\section{Theory II: Literature review}

- Combining Banfield (1958) and Coleman (1988) arguments,

- according to which stem family types are an important determinant of contemporaneous levels of social capital 


\section{Theory II: Literature review}

- Combining Banfield (1958) and Coleman (1988) arguments,

- according to which stem family types are an important determinant of contemporaneous levels of social capital

- With the Emmanuel Todd (1985) arguments that historical family types affect not only development but also social and political hierarchies: 


\section{Theory II: Literature review}

- Combining Banfield (1958) and Coleman (1988) arguments,

- according to which stem family types are an important determinant of contemporaneous levels of social capital

- With the Emmanuel Todd (1985) arguments that historical family types affect not only development but also social and political hierarchies:

- and, specifically, stem family types are associated with greater out-group trust, political trust and cooperation 


\section{Theory II: Literature review}

- Combining Banfield (1958) and Coleman (1988) arguments,

- according to which stem family types are an important determinant of contemporaneous levels of social capital

- With the Emmanuel Todd (1985) arguments that historical family types affect not only development but also social and political hierarchies:

- and, specifically, stem family types are associated with greater out-group trust, political trust and cooperation

- and, also, that contemporaneous levels of social capital and it's ability to generate public goods might depend on the vertical and horizontal structure of organizations (Boix and Posner 1998) 


\section{Theory III: The argument}

- Higher social capital in stem family areas, with a denser network of societal relations, might be an important insurance mechanism against unemployment losses: 


\section{Theory III: The argument}

- Higher social capital in stem family areas, with a denser network of societal relations, might be an important insurance mechanism against unemployment losses:

- Stem historical families: non-egalitarian and strong authority families (no equal inheritance, no cohabitation) 


\section{Theory III: The argument}

- Higher social capital in stem family areas, with a denser network of societal relations, might be an important insurance mechanism against unemployment losses:

- Stem historical families: non-egalitarian and strong authority families (no equal inheritance, no cohabitation)

- Communitarian historical families: egalitarian and strong authority families (equal inheritance, cohabitation) 


\section{Theory III: The argument}

- Higher social capital in stem family areas, with a denser network of societal relations, might be an important insurance mechanism against unemployment losses:

- Stem historical families: non-egalitarian and strong authority families (no equal inheritance, no cohabitation)

- Communitarian historical families: egalitarian and strong authority families (equal inheritance, cohabitation)

- In a reduced form framework, and given persistence of the effects of family types, we should observe lower political backlash with current unemployment shocks when there is a dense network of social relations to rely upon 


\section{Theory IV: The argument}

- Given a contemporaneous unemployment shock with job losses, historical family types should moderate the effects:

1 Greater social capital in stem family areas should act as an insurance mechanism preventing political backlash 


\section{Theory IV: The argument}

- Given a contemporaneous unemployment shock with job losses, historical family types should moderate the effects:

1 Greater social capital in stem family areas should act as an insurance mechanism preventing political backlash

2 At the same time, contemporaneous levels of social capital in stem family areas might be eroded by the negative income shock 


\section{Theory IV: The argument}

- Given a contemporaneous unemployment shock with job losses, historical family types should moderate the effects:

1 Greater social capital in stem family areas should act as an insurance mechanism preventing political backlash

2 At the same time, contemporaneous levels of social capital in stem family areas might be eroded by the negative income shock

3 In communitarian family areas, the unemployment losses can be associated with more political backslash, trust and nationalist voting 


\section{Theory IV: The argument}

- Given a contemporaneous unemployment shock with job losses, historical family types should moderate the effects:

1 Greater social capital in stem family areas should act as an insurance mechanism preventing political backlash

2 At the same time, contemporaneous levels of social capital in stem family areas might be eroded by the negative income shock

3 In communitarian family areas, the unemployment losses can be associated with more political backslash, trust and nationalist voting

4 The net total effect could imply a reversal of the effect of social capital on political backlash and nationalist voting 


\section{Hypotheses}

Given an unemployment shock, stem family regions should reduce the extent of political backlash via nationalist voting, because of the persistence of high levels of social capital $(\mathrm{H} 1)$ 


\section{Hypotheses}

Given an unemployment shock, stem family regions should reduce the extent of political backlash via nationalist voting, because of the persistence of high levels of social capital $(\mathrm{H} 1)$

Given an unemployment shocks, communitarian family regions should amplify political backlash via nationalist voting, because of the persistence of low levels of social capital $(\mathrm{H} 2)$ 


\section{Hypotheses}

Given an unemployment shock, stem family regions should reduce the extent of political backlash via nationalist voting, because of the persistence of high levels of social capital $(\mathrm{H} 1)$

Given an unemployment shocks, communitarian family regions should amplify political backlash via nationalist voting, because of the persistence of low levels of social capital $(\mathrm{H} 2)$ 


\section{Data collection I}

Individual survey-data matched with European regional data

- ESS Surveys

- New dataset on job layoffs, European Restructuring Monitor (ERM)

- Emmanuel Todd data on historical family types

- Social capital indicators at the regional-level:

- ESS (pre-shock data, early 2000s)

- EVS (pre-shock and post-sock data, comparable measures)

- EU-SILC (pre-shock and post-shock data) 


\section{Data collection II: Layoffs data by Restructuring Monitor}

- Rich firm-level data covering 2002-2018, we calculate the average of the ratio of layoffs by region and ESS round

- It records restructurings which involve the creation or destruction of at least 100 jobs, or affect $10 \%$ of the workforce at sites employing more than 250 people

- First, we calculate the ratio between the announced direct dismissals and the number of employees (at the firm-level) for each of the observation in ERM data

- Second, we calculate the total by region (NUTS 2, NUTS1) and announcement year (coded from news in newspapers)

- Then, we match the announcement year with the corresponding ESS round to calculate totals by region and ESS round, and finally, the average 


\section{Data collection III: Social Capital at the Regional Level}

- Data Pre-shock: using the module on Citizens Involvement from ESS Round 1

- We calculate the regional average of social capital based on the membership or participation of the respondent in at least one of the following organizations:

- sports, cultural, humanitarian, environmental, science, social club, or voluntary organization

- We create the dummy which takes the value of 1 if the respondent belongs to at least one of the aforementioned organizations, and 0 otherwise

- Then, we generate the variable mean social capital calculating the regional averages 


\section{Data collection IV: Social Capital at the Regional Level}

- Data pre and post shock: using data from the European Values Study (EVS) from 1981 to 2010

- We calculate the regional average of social capital based on the membership or voluntary work of the respondent in at least one of the following organizations:

- cultural, human rights, conservation of environment and animals, conservation of environment, animal rights, youth, sports, or health

- We generate the variable social capital evs which takes the value of 1 if the respondent belongs to at least one of the organizations, 0 otherwise

- Then, calculating the regional average of social capital evs 


\section{Empirical strategy}

Combination of different strategies

1 Individual-level analysis

2 Regional-level analysis, before and after

3 Cross-sectional regional evidence to explore the mechanism 


\section{Roadmap}

1 Exploration of the shock: Unemployment shock, job dismissals

2 DVs: Nationalism scores (as in Colantone and Stanig), trust in politicians, inter-personal trust

3 Individual-level baseline models first, afterwards regional-level

4 Exploring the mechanism: social capital 


\section{Historical family types across European regions}

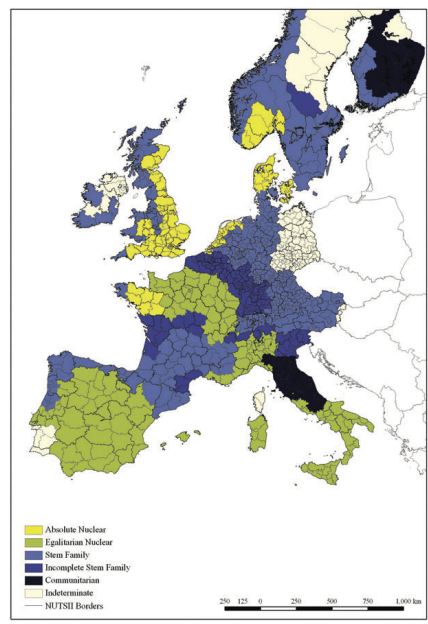




\section{Econometric specification:}

- Individual-level specifications:

$$
\begin{array}{r}
Y_{i j t}=\beta_{1} \text { UnemplShock }_{j t}+\beta_{2} \text { HistoricalFamily }_{j}+ \\
+\gamma \text { UnempShock }_{j t} \times \text { HistoricalFamily }_{j}+ \\
+\delta_{1} X_{i}+\delta_{2} R_{j t}+\delta_{3} C Y+\varepsilon_{i j}
\end{array}
$$

- All models include Country X Year FEs

- Standard errors are always clustered at the regional-level 


\section{Unemployment shock and Nationalism: Stem families}

\begin{tabular}{|c|c|c|c|}
\hline & $\begin{array}{c}\text { Nationalism } \\
\text { Score }\end{array}$ & $\begin{array}{c}\text { (2) } \\
\text { Nationalism } \\
\text { Score }\end{array}$ & $\begin{array}{c}\text { (3) } \\
\text { Nationalism } \\
\text { Score }\end{array}$ \\
\hline Stem & $\begin{array}{c}0.292^{* * *} \\
(0.083)\end{array}$ & $\begin{array}{c}0.328^{* * *} \\
(0.092)\end{array}$ & $\begin{array}{c}0.319 * * * \\
(0.091)\end{array}$ \\
\hline Unemployment & $\begin{array}{c}-0.012 * * \\
(0.006)\end{array}$ & $\begin{array}{c}-0.021 * * * \\
(0.006)\end{array}$ & $\begin{array}{c}-0.019 * * * \\
(0.005)\end{array}$ \\
\hline Stem X Unempl & $\begin{array}{c}-0.048 * * * \\
(0.015) \\
\end{array}$ & $\begin{array}{c}-0.051^{* * *} \\
(0.014)\end{array}$ & $\begin{array}{c}-0.051 * * * \\
(0.014)\end{array}$ \\
\hline Individual Controls & No & No & Yes \\
\hline Regional Controls & No & Yes & Yes \\
\hline CountryXYear FEs & Yes & Yes & Yes \\
\hline ESS Rounds FEs & Yes & Yes & Yes \\
\hline Mean Dep. Var & 1.665 & 1.604 & 1.604 \\
\hline$N$ & 78967 & 69751 & 69537 \\
\hline Number of regions & 136 & 124 & 124 \\
\hline Number of countries & 12 & 11 & 11 \\
\hline
\end{tabular}

Standard errors clustered at the regional level in parentheses. Individual Controls: Gender, age, education.

Regional Controls: Log regional GDP $* p<0.10, * * p<0.05, * * * p<0.01$. 


\section{Unemployment shock and Nationalism: Communitarian}

\begin{tabular}{lccc}
\hline \hline & $(1)$ & $(2)$ & $(3)$ \\
& Nationalism & Nationalism & Nationalism \\
& Score & Score & Score \\
\hline Communitarian & $-1.271^{* * *}$ & $-1.373^{* * *}$ & $\left(0.374^{* * *}\right.$ \\
& $(0.353)$ & $(0.329)$ & $-0.023^{* * *}$ \\
& & & $(0.007)$ \\
& $-0.015^{* *}$ & $-0.025^{* * *}$ & $(0.007)$ \\
Unemployment & $(0.007)$ & & $0.132^{* * *}$ \\
& & $0.131^{* * *}$ & $(0.035)$ \\
\hline Individual Controls & $0.121^{* * *}$ & $(0.034)$ & Yes \\
Regional Controls & $(0.038)$ & No & Yes \\
CountryXYear FEs & No & Yes & Yes \\
ESS Rounds FEs & Yes & Yes & 1.604 \\
Mean Dep. Var & 1.665 & 1.604 & 69537 \\
$N$ & 78967 & 69751 & 124 \\
Number of regions & 136 & 124 & 11 \\
Number of countries & 12 & 11 & Yes \\
\hline \hline
\end{tabular}

Standard errors clustered at the regional level in parentheses. Individual Controls: Gender, age, education.

Regional Controls: Log regional GDP $* p<0.10, * * p<0.05, * * * p<0.01$. 


\section{Job dismissals and Nationalism: Stem families}

\begin{tabular}{lccc}
\hline & $(1)$ & $(2)$ & $(3)$ \\
& Nationalism & Nationalism & Nationalism \\
Score & Score & -0.013 \\
& 0.025 & -0.009 & $(0.054)$ \\
& $(0.046)$ & $(0.056)$ & $0.003^{*}$ \\
Dismissals & 0.002 & & $(0.002)$ \\
& $(0.002)$ & 0.003 & \\
& & $(0.002)$ & $-0.007^{*}$ \\
Stem X Dismissals & -0.005 & $-0.007^{*}$ & $(0.004)$ \\
& $(0.003)$ & $(0.004)$ & Yes \\
Individual Controls & No & No & Yes \\
Regional Controls & No & Yes & Yes \\
CountryXYear FEs & Yes & Yes & Yes \\
ESS Rounds FEs & Yes & Yes & 1.606 \\
Mean Dep. Var & 1.609 & 1.606 & 66589 \\
$N$ & 90792 & 66799 & 122 \\
Number of regions & 136 & 122 & 11 \\
Number of countries & 13 & 11 & (13) \\
\hline
\end{tabular}

Standard errors clustered at the regional level in parentheses. Individual Controls: Gender, age, education. Regional Controls: Log regional GDP, regional unemployment $* p<0.10, * * p<0.05, * * * p<0.01$. 


\section{Job dismissals and Nationalism: Communitarian}

\begin{tabular}{lccc}
\hline \hline & $\begin{array}{c}(1) \\
\text { Nationalism } \\
\text { Score }\end{array}$ & $\begin{array}{c}(2) \\
\text { Nationalism } \\
\text { Score }\end{array}$ & $\begin{array}{c}(3) \\
\text { Nationalism } \\
\text { Score }\end{array}$ \\
\hline Communitarian & $-0.287^{* * *}$ & $-0.454^{* * *}$ & $-0.456^{* * *}$ \\
& $(0.106)$ & $(0.141)$ & $(0.144)$ \\
Dismissals & -0.001 & -0.002 & -0.002 \\
& $(0.001)$ & $(0.002)$ & $(0.002)$ \\
Commun X Dismissals & 0.004 & & \\
& $(0.003)$ & $0.022^{*}$ & $0.024^{* *}$ \\
Individual Controls & No & $(0.011)$ & $(0.010)$ \\
Regional Controls & No & No & Yes \\
CountryXYear FEs & Yes & Yes & Yes \\
ESS Rounds FEs & Yes & Yes & Yes \\
Mean Dep. Var & 1.609 & 1.606 & Yes \\
$N$ & 90792 & 66799 & 1.606 \\
Number of regions & 136 & 122 & 66589 \\
Number of countries & 13 & 11 & 122 \\
\hline
\end{tabular}

Standard errors clustered at the regional level in parentheses. Individual Controls: Gender, age, education. Regional Controls: Log regional GDP, regional unemployment $* p<0.10, * * p<0.05, * * * p<0.01$. 


\section{Unemployment shock and Nationalism}

Marginal Effects of Unemployment on Nationalism Score: Conditional on Family Types

Stem Families

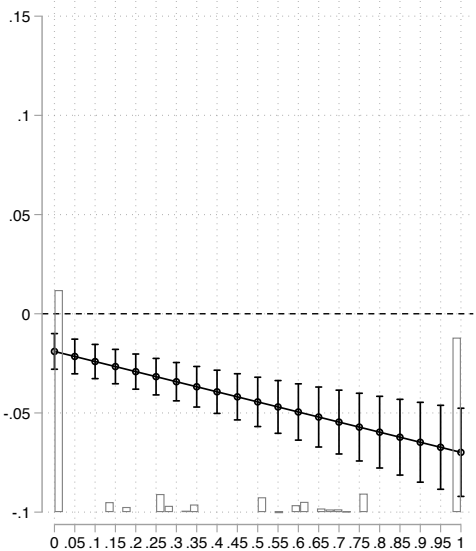

Communitarian Families

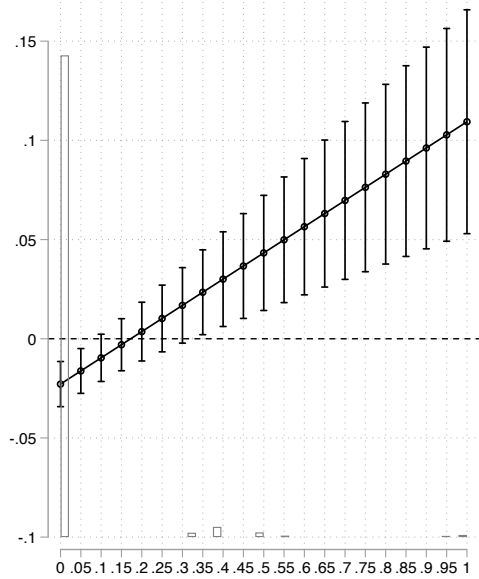




\section{Job dismissals shock and Nationalism}

Marginal Effects of Job Dismissals on Nationalism Score: Conditional on Family Types
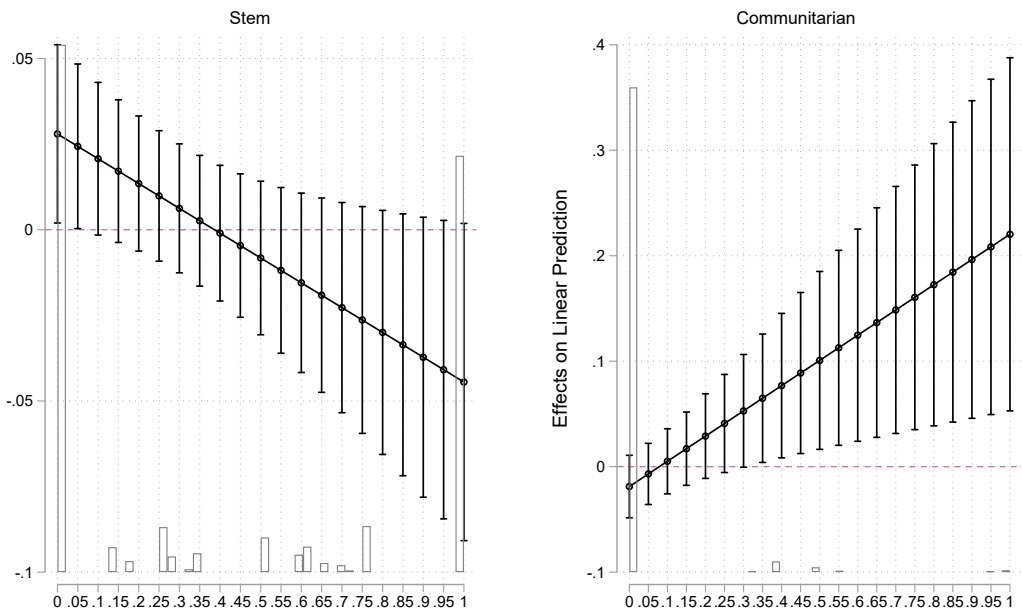

Amat, Francesc

Democracy in Hard Times

APSA 2020 


\section{Job dismissals shock and Political Trust: Stem families}

\begin{tabular}{|c|c|c|c|}
\hline & $\begin{array}{c}\text { (1) } \\
\text { Trust in } \\
\text { Politicians }\end{array}$ & $\begin{array}{c}\text { (2) } \\
\text { Trust in } \\
\text { Politicians }\end{array}$ & $\begin{array}{c}\text { (3) } \\
\text { Trust in } \\
\text { Politicians }\end{array}$ \\
\hline Stem & $\begin{array}{l}0.092^{* *} \\
(0.047)\end{array}$ & $\begin{array}{c}0.071 \\
(0.053)\end{array}$ & $\begin{array}{c}0.080 \\
(0.053)\end{array}$ \\
\hline Dismissals & $\begin{array}{c}0.007 * * * \\
(0.002)\end{array}$ & $\begin{array}{l}0.004 * * \\
(0.002)\end{array}$ & $\begin{array}{l}0.004 * * \\
(0.002)\end{array}$ \\
\hline Stem X Dismissals & $\begin{array}{c}-0.008 * * * \\
(0.003)\end{array}$ & $\begin{array}{c}-0.006 * * * \\
(0.002)\end{array}$ & $\begin{array}{c}-0.006 * * * \\
(0.002)\end{array}$ \\
\hline Individual Controls & No & No & Yes \\
\hline Regional Controls & No & Yes & Yes \\
\hline CountryXYear FEs & Yes & Yes & Yes \\
\hline ESS Rounds FEs & Yes & Yes & Yes \\
\hline Mean Dep. Var & 3.753 & 3.560 & 3.562 \\
\hline$N$ & 151708 & 114062 & 113428 \\
\hline Number of regions & 136 & 122 & 122 \\
\hline Number of countries & 13 & 11 & 11 \\
\hline
\end{tabular}

Standard errors clustered at the regional level in parentheses. Individual Controls: Gender, age, education. Regional Controls: Log regional GDP, regional unemployment $* p<0.10, * * p<0.05, * * * p<0.01$. 


\section{Job dismissals and Political Trust: Communitarian}

\begin{tabular}{lccc}
\hline \hline & $\begin{array}{c}(1) \\
\text { Trust in } \\
\text { Politicians }\end{array}$ & $\begin{array}{c}(2) \\
\text { Trust in } \\
\text { Politicians }\end{array}$ & $\begin{array}{c}\text { Trust in } \\
\text { Politicians }\end{array}$ \\
\hline Communitarian & 0.037 & -0.295 & -0.272 \\
& $(0.283)$ & $(0.201)$ & $(0.204)$ \\
Dismissals & 0.002 & & -0.000 \\
& $(0.001)$ & $(0.001)$ & $(0.001)$ \\
Commun X Dismissals & $0.032^{* *}$ & $0.048^{* * *}$ & \\
& $(0.013)$ & $(0.013)$ & $0.047^{* * *}$ \\
Individual Controls & No & No & $(0.012)$ \\
Regional Controls & No & Yes & Yes \\
CountryXYear FEs & Yes & Yes & Yes \\
ESS Rounds FEs & Yes & Yes & Yes \\
Mean Dep. Var & 3.753 & 3.560 & 3.562 \\
$N$ & 151708 & 114062 & 113428 \\
Number of regions & 136 & 122 & 122 \\
Number of countries & 13 & 11 & 11 \\
\hline
\end{tabular}

Standard errors clustered at the regional level in parentheses. Individual Controls: Gender, age, education. Regional Controls: Log regional GDP, regional unemployment $* p<0.10, * * p<0.05, * * * p<0.01$. 


\section{Job dismissals shock and Political Trust}

Marginal Effects of Job Dismissals on Trust in Politicians: Conditional on Family Types
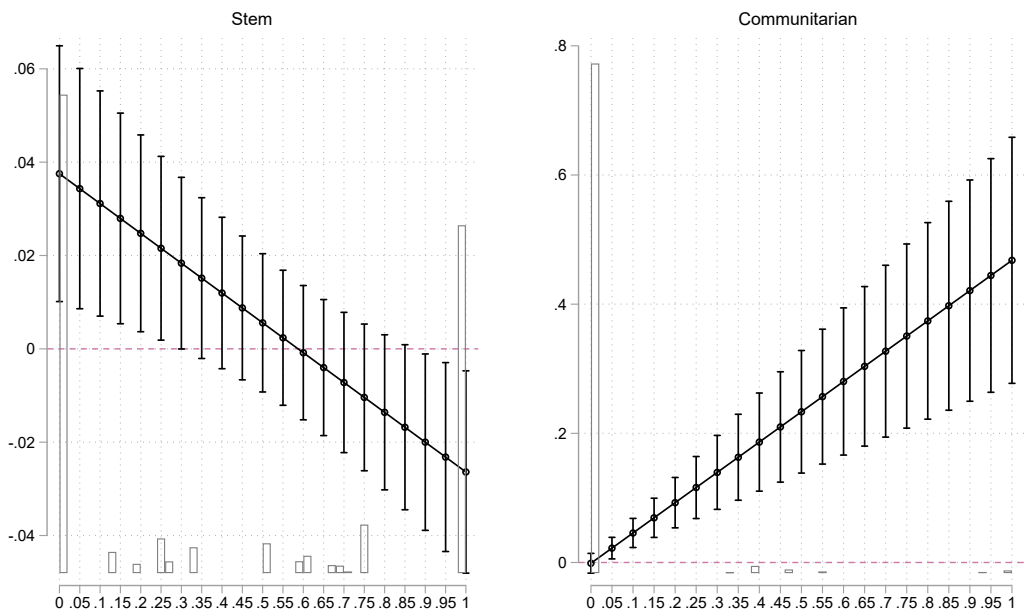


\section{Regional Level-Analysis I: Nationalism}

\begin{tabular}{|c|c|c|c|c|}
\hline & $\begin{array}{c}(1) \\
\text { Nationalism } \\
\text { Score }\end{array}$ & $\begin{array}{c}(2) \\
\text { Nationalism } \\
\text { Score }\end{array}$ & $\begin{array}{c}(3) \\
\text { Nationalism } \\
\text { Score }\end{array}$ & $\begin{array}{l}(4) \\
\text { Nationalism } \\
\text { Score }\end{array}$ \\
\hline Unemployment Post & $\begin{array}{l}-0.001 \\
(0.005)\end{array}$ & $\begin{array}{l}0.019 * * \\
(0.007)\end{array}$ & $\begin{array}{c}0.001 \\
(0.006)\end{array}$ & $\begin{array}{c}0.027^{* * *} \\
(0.009)\end{array}$ \\
\hline Stem & $\begin{array}{c}0.217 * * \\
(0.092)\end{array}$ & $\begin{array}{c}0.219 * * * \\
(0.062)\end{array}$ & & \\
\hline UnemplPost $X$ Stem & $\begin{array}{c}-0.034 * * * \\
(0.008)\end{array}$ & $\begin{array}{c}-0.033^{* * *} \\
(0.005)\end{array}$ & & \\
\hline Communitarian & & & $\begin{array}{c}-1.062^{* * *} \\
(0.200)\end{array}$ & $\begin{array}{c}-1.525 * * * \\
(0.319)\end{array}$ \\
\hline UnemplPost X Commun & & & $\begin{array}{c}0.105 * * * \\
(0.020)\end{array}$ & $\begin{array}{c}0.129 * * * \\
(0.031)\end{array}$ \\
\hline Log GDP pc Pre & $\begin{array}{c}-0.158 \\
(0.110)\end{array}$ & $\begin{array}{c}-0.290 * * \\
(0.123)\end{array}$ & $\begin{array}{c}-0.148 \\
(0.107)\end{array}$ & $\begin{array}{c}-0.329 * * \\
(0.137)\end{array}$ \\
\hline Unemployment Pre & & $\begin{array}{c}-0.028 * * * \\
(0.004)\end{array}$ & & $\begin{array}{c}-0.038^{* * *} \\
(0.010)\end{array}$ \\
\hline Country FEs & Yes & Yes & Yes & Yes \\
\hline Mean Dep. Var & 1.607 & 1.647 & 1.607 & 1.647 \\
\hline$R^{2}$ & 0.832 & 0.869 & 0.818 & 0.861 \\
\hline Number of regions & 136 & 121 & 136 & 121 \\
\hline Number of countries & 12 & 12 & 12 & 12 \\
\hline
\end{tabular}

Standard errors clustered at the country level in parentheses. 


\section{Regional Level-Analysis II: Trust in Politicians}

\begin{tabular}{|c|c|c|c|c|}
\hline Unemployment Post & $\begin{array}{c}\text { (1) } \\
\begin{array}{c}\text { Trust in } \\
\text { Politicians }\end{array} \\
0.011 \\
(0.013)\end{array}$ & $\begin{array}{c}(2) \\
\begin{array}{c}\text { Trust in } \\
\text { Politicians }\end{array} \\
0.005 \\
(0.010)\end{array}$ & $\begin{array}{c}\text { (3) } \\
\begin{array}{c}\text { Trust in } \\
\text { Politicians }\end{array} \\
0.013 \\
(0.018)\end{array}$ & $\begin{array}{c}\begin{array}{c}(4) \\
\text { Trust in } \\
\text { Politicians }\end{array} \\
0.002 \\
(0.016)\end{array}$ \\
\hline Stem & $\begin{array}{l}0.173 * \\
(0.080)\end{array}$ & $\begin{array}{c}0.166 * * * \\
(0.052)\end{array}$ & & \\
\hline UnemplPost X Stem & $\begin{array}{c}-0.018^{* * *} \\
(0.006)\end{array}$ & $\begin{array}{c}-0.015 * * * \\
(0.004)\end{array}$ & & \\
\hline Communitarian & & & $\begin{array}{l}-0.560 \\
(0.639)\end{array}$ & $\begin{array}{c}-0.903^{* *} \\
(0.390)\end{array}$ \\
\hline UnempIPost X Commun & & & $\begin{array}{c}0.064 \\
(0.063)\end{array}$ & $\begin{array}{c}0.119 * * \\
(0.041)\end{array}$ \\
\hline Trust in Politicians Pre & & $\begin{array}{c}0.337 * * * \\
(0.059)\end{array}$ & & $\begin{array}{c}0.387 * * * \\
(0.061)\end{array}$ \\
\hline Log GDP pc Pre & $\begin{array}{c}0.594 * * \\
(0.193)\end{array}$ & $\begin{array}{c}0.461^{* *} \\
(0.179)\end{array}$ & $\begin{array}{l}0.599 * * \\
(0.194)\end{array}$ & $\begin{array}{c}0.460 * * \\
(0.180)\end{array}$ \\
\hline Unemployment Pre & $\begin{array}{c}-0.003 \\
(0.015)\end{array}$ & $\begin{array}{c}0.004 \\
(0.012)\end{array}$ & $\begin{array}{l}-0.004 \\
(0.020)\end{array}$ & $\begin{array}{c}0.009 \\
(0.021)\end{array}$ \\
\hline $\begin{array}{l}\text { Country FEs } \\
\text { Mean Dep. Var } \\
R^{2} \\
\text { Number of regions } \\
\text { Number of countries }\end{array}$ & $\begin{array}{l}\text { Yes } \\
3.324 \\
0.953 \\
121 \\
12 \\
\end{array}$ & $\begin{array}{c}\text { Yes } \\
3.324 \\
0.961 \\
121 \\
12 \\
\end{array}$ & $\begin{array}{c}\text { Yes } \\
3.324 \\
0.952 \\
121 \\
12 \\
\end{array}$ & $\begin{array}{l}\text { Yes } \\
3.324 \\
0.962 \\
121 \\
12 \\
\end{array}$ \\
\hline
\end{tabular}

Standard errors clustered at the country level in parentheses.

$* p<0.10$, ** $p<0.05$, *** $p<0.01$. 


\section{Exploring the Mechanism I: Social Capital}

\begin{tabular}{|c|c|c|c|c|}
\hline & $\begin{array}{l}\text { Social Capital } \\
\text { Pre }\end{array}$ & $\begin{array}{l}\text { Social Capital } \\
\text { Pre }\end{array}$ & $\begin{array}{l}\text { Social Capital } \\
\text { Pre }\end{array}$ & $\begin{array}{l}\text { Social Capital } \\
\text { Pre }\end{array}$ \\
\hline Stem & $\begin{array}{c}0.097^{* * *} \\
(0.030)\end{array}$ & $\begin{array}{c}0.057^{* *} \\
(0.024)\end{array}$ & $\begin{array}{l}0.032^{*} \\
(0.018)\end{array}$ & \\
\hline Communitarian & $\begin{array}{c}-0.131 * * \\
(0.055)\end{array}$ & $\begin{array}{c}-0.162 * * \\
(0.074)\end{array}$ & & $\begin{array}{c}-0.127 * \\
(0.071)\end{array}$ \\
\hline Log GDP pc Pre & & $\begin{array}{c}0.317 * * * \\
(0.037)\end{array}$ & $\begin{array}{c}0.055 \\
(0.039)\end{array}$ & $\begin{array}{c}0.042 \\
(0.035)\end{array}$ \\
\hline Unemployment Pre & & $\begin{array}{l}-0.005^{*} \\
(0.003)\end{array}$ & $\begin{array}{c}-0.006^{* *} \\
(0.002)\end{array}$ & $\begin{array}{c}-0.009 * * * \\
(0.002)\end{array}$ \\
\hline $\begin{array}{l}\text { Country FEs } \\
\text { Mean Dep. Var }\end{array}$ & $\begin{array}{l}\text { No } \\
0.537\end{array}$ & $\begin{array}{l}\text { No } \\
0.530\end{array}$ & $\begin{array}{l}\text { Yes } \\
0.530\end{array}$ & $\begin{array}{l}\text { Yes } \\
0.530\end{array}$ \\
\hline $\begin{array}{l}R^{2} \\
\text { Number of regions } \\
\text { Number of countries }\end{array}$ & $\begin{array}{c}0.101 \\
136 \\
13\end{array}$ & $\begin{array}{c}0.591 \\
120 \\
12\end{array}$ & $\begin{array}{c}0.836 \\
120 \\
12\end{array}$ & $\begin{array}{c}0.842 \\
120 \\
12\end{array}$ \\
\hline
\end{tabular}

Standard errors in parentheses.

* $p<0.10$, ** $p<0.05, * * * p<0.01$. 


\section{Exploring the Mechanism II: Social Capital}

\begin{tabular}{|c|c|c|c|c|}
\hline Unemployment Post & $\begin{array}{c}\text { Social Capital } \\
\text { Post } \\
-0.023^{* * *} \\
(0.003)\end{array}$ & $\begin{array}{c}\text { Social Capital } \\
\text { Post } \\
-0.002 \\
(0.003)\end{array}$ & $\begin{array}{c}\text { (3) } \\
\text { Social Capital } \\
\text { Post } \\
-0.031^{* * *} \\
(0.006)\end{array}$ & $\begin{array}{c}\text { Social Capital } \\
\text { Post } \\
-0.010^{* *} \\
(0.005)\end{array}$ \\
\hline Communitarian & $\begin{array}{c}-0.780 * * * \\
(0.238)\end{array}$ & $\begin{array}{l}-0.188 \\
(0.140)\end{array}$ & & \\
\hline UnemplPost X Commun & $\begin{array}{c}0.070 * * * \\
(0.020)\end{array}$ & $\begin{array}{l}0.022 * \\
(0.012)\end{array}$ & & \\
\hline Egalitarian Nuclear & & & $\begin{array}{c}-0.333^{* * *} \\
(0.072)\end{array}$ & $\begin{array}{c}-0.174 * \\
(0.088)\end{array}$ \\
\hline UnemplPost X Egalitarian & & & $\begin{array}{c}0.023 * * * \\
(0.006)\end{array}$ & $\begin{array}{l}0.014 * * \\
(0.006)\end{array}$ \\
\hline Social Capital Pre & & $\begin{array}{c}1.114^{* * *} \\
(0.105)\end{array}$ & & $\begin{array}{c}1.071 * * * \\
(0.104)\end{array}$ \\
\hline $\begin{array}{l}\text { Mean Dep. Var } \\
R^{2} \\
\text { Number of regions } \\
\text { Number of countries }\end{array}$ & $\begin{array}{l}0.344 \\
0.313 \\
126 \\
12\end{array}$ & $\begin{array}{l}0.356 \\
0.668 \\
106 \\
12\end{array}$ & $\begin{array}{c}0.344 \\
0.370 \\
126 \\
12\end{array}$ & $\begin{array}{l}0.356 \\
0.683 \\
106 \\
12\end{array}$ \\
\hline
\end{tabular}

Standard errors in parentheses.

* $p<0.10$, ** $p<0.05$, *** $p<0.01$. 


\section{Exploring the Mechanism III: Social Capital Reversal}

\begin{tabular}{lccc}
\hline & Nationalism & Nationalism & Nationalism \\
& Score & Score & Score \\
\hline Social Capital Pre & $-0.775^{* *}$ & $-0.712^{* *}$ & $-0.682^{*}$ \\
& $(0.341)$ & $(0.330)$ & $(0.372)$ \\
\hline Individual Controls & No & Yes & Yes \\
Regional Controls & No & No & Yes \\
CountryXYear FEs & Yes & Yes & Yes \\
ESS Rounds FEs & Yes & Yes & Yes \\
Mean Dep. Var & 1.624 & 1.624 & 1.635 \\
$N$ & 130756 & 130206 & 96822 \\
Number of regions & 187 & 187 & 156 \\
Number of countries & 18 & 18 & 15 \\
\hline \hline
\end{tabular}

Standard errors clustered at the regional level in parentheses.

Individual Controls: Gender, age, education.

Regional Controls: Log regional GDP

$* p<0.10, * * p<0.05, * * * p<0.01$. 


\section{Exploring the Mechanism IV: Social Capital Reversal}

\begin{tabular}{lcccc}
\hline \hline & $\begin{array}{c}\text { Nationalism } \\
\text { Score }\end{array}$ & $\begin{array}{c}\text { Nationalism } \\
\text { Score }\end{array}$ & $\begin{array}{c}\text { Nationalism } \\
\text { Score }\end{array}$ & $\begin{array}{c}\text { Nationalism } \\
\text { Score }\end{array}$ \\
\hline Social Capital Pre & -0.478 & $-0.952^{*}$ & & \\
& $(0.385)$ & $(0.534)$ & & \\
Social Capital Post & & & $0.441^{* *}$ & $0.432^{* *}$ \\
& & & $0.209)$ & Yes \\
\hline Individual Controls & No & Yes & No & Yes \\
Regional Controls & Yes & Yes & Yes & Yes \\
CountryXYear FEs & Yes & Yes & 1.54 & 1.617 \\
ESS Rounds FEs & 1.940 & 1.932 & 64075 & 17658 \\
Mean Dep. Var & 66120 & 44722 & 195 & 157 \\
$N$ & 149 & 115 & 23 & 19 \\
Number of regions & 20 & 16 & \\
Number of countries & & & & \\
\hline \hline
\end{tabular}

Standard errors clustered at the regional level in parentheses.

Individual Controls: Gender, age, education.

Regional Controls: Log regional GDP, regional unemployment

$* p<0.10, * * p<0.05$, *** $p<0.01$. 


\section{Conclusions}

The main findings are: 


\section{Conclusions}

The main findings are:

- Historical family types moderate the effect of contemporaneous unemployment shocks on voting patterns and political trust 


\section{Conclusions}

The main findings are:

- Historical family types moderate the effect of contemporaneous unemployment shocks on voting patterns and political trust

- Stem family regions alleviate shocks, whereas communitarian family regions amplify the political backlash 


\section{Conclusions}

The main findings are:

- Historical family types moderate the effect of contemporaneous unemployment shocks on voting patterns and political trust

- Stem family regions alleviate shocks, whereas communitarian family regions amplify the political backlash

- Social capital as the mediating factor linking historical family types and contemporaneous shocks and political responses 


\section{Conclusions}

The main findings are:

- Historical family types moderate the effect of contemporaneous unemployment shocks on voting patterns and political trust

- Stem family regions alleviate shocks, whereas communitarian family regions amplify the political backlash

- Social capital as the mediating factor linking historical family types and contemporaneous shocks and political responses

- Social capital reverses the effect before and after the economic crisis 


\section{Implications}

The findings suggest that social capital is a mediating variable: 


\section{Implications}

The findings suggest that social capital is a mediating variable:

- Reversal of the effect of social capital on nationalism voting, before and after the 2008 economic crisis

- Potential reconciliation of the literature: social capital itself as a function of contemporaneous economic shocks and deep and pre-existing cultural roots 